\title{
Influencia del acompañamiento pedagógico en el desempeño docente en la unidad de gestión educativa local de Santiago de Chuco, 2020
}

\author{
Marian Ella Del Milagro Chávez Otero \\ marianchavezotero@gmail.com \\ Orcid: https://orcid.org/0000-0002-6511-5779 \\ Formador-Tutor multigrado-UGEL Santiago de Chuco \\ Dulio Oseda Gago \\ dosedag@unmsm.edu.pe \\ Orcid: https://orcid.org/0000-0002-3136-6094 \\ Universidad Nacional Mayor de San Marcos
}

\section{RESUMEN}

El presente estudio se orientó con el objetivo de determinar la influencia del acompañamiento pedagógico en el desempeño docente en la Unidad de Gestión Educativa Local de Santiago de Chuco.

Por otro lado, el estudio fue de enfoque cuantitativo. Presenta un diseño no experimental transversal descriptivo correlacional causal. La técnica fue utilizada fue la encuesta y los instrumentos fueron dos cuestionarios con escala de Likert. La muestra estuvo constituida por 24 docentes.

Finalmente, los hallazgos determinaron que, en relación a la prueba de hipótesis general, el estadístico de Rho de Spearman, demuestra que, (p-valor: 0,183>0,010), en consecuencia, se acepta la hipótesis nula (Ho) y se rechaza la hipótesis alterna (Hi), se concluye que, el acompañamiento pedagógico no influye significativamente en el desempeño docente en la Unidad de Gestión Educativa Local de Santiago de Chuco, 2020.

Palabras clave: acompañamiento pedagógico; desempeño docente; enseñanza; aprendizaje; evaluación. 


\title{
Influence of pedagogical accompaniment on teaching performance in
} the local educational management unit of Santiago de Chuco, 2020

\begin{abstract}
The present study was oriented with the objective of determining the influence of pedagogical accompaniment on teaching performance in the Local Educational Management Unit of Santiago de Chuco.

On the other hand, the study had a quantitative approach. It presents a non-experimental cross-sectional descriptive correlational causal design. The technique used was the survey and the instruments were two questionnaires with a Likert scale. The sample consisted of 24 teachers.

Finally, the findings determined that, in relation to the general hypothesis test, the Spearman Rho statistic shows that, (p-value: 0.183>0.010), consequently, the null hypothesis (Ho) is accepted and rejected the alternate hypothesis (Hi), it is concluded that the pedagogical accompaniment does not significantly influence the teaching performance in the Local Educational Management Unit of Santiago de Chuco, 2020.
\end{abstract}

Keywords: pedagogical support; teacher performance; teaching; learning; evaluation.

Artículo recibido: 10. Junio. 2021 Aceptado para publicación: 16. Julio. 2021 Correspondencia: dosedag@unmsm.edu.pe Conflictos de Interés: Ninguna que declarar 


\section{INTRODUCCIÓN}

La calidad de la Educación, es un compromiso que han asumido los Estados y las instituciones, lo cual demuestra que, existen claras intenciones de mejorar las condiciones en el proceso de planificación, asociada a la enseñanza y el aprendizaje de los estudiantes. En esa intención la función que desempeña el docente es fundamental, sin embargo, necesita de la asistencia técnica y acompañamiento para reforzar sus competencias pedagógicas (Cuenca, 2015).

La problemática, del presente estudio, se visualiza en diversos ámbitos, tal es así que en el ámbito internacional, la Organización de las Naciones Unidas para la Educación, la Ciencia y la Cultura, (UNESCO, 2019), sostiene que, la calidad de la Educación se garantiza con la calidad de la enseñanza y el trabajo del docente, es decir que, el éxito de la Educación se centra en la labor docente, porque se puede contar con la infraestructura y los recursos necesarios, así como carecer de ellos, pero en ambas situaciones se impone el trabajo que desarrolla el docente, tanto en una planificación pertinente como en la ejecución de lo planificado, pero que responda a las necesidades de aprendizaje de los estudiantes, lo cual repercute en el rendimiento académico.

Así mismo, en América Latina, el bajo rendimiento de los estudiantes ha generado un replanteamiento en las políticas educativas para revertir esa realidad. Los programas de formación en servicio docente, se promueven sin realizar un verdadero diagnóstico en el desempeño pedagógico de los docentes, lo cual, no responde a las verdaderas necesidades de la práctica pedagógica, por otro lado, la reducida escala salarial, además de una carencia en el reconocimiento e incentivo para la mejora continua, genera como consecuencia, una desmotivación y descompromiso por la carrera docente (Rivas, 2015). En el Perú, la implementación del acompañamiento pedagógico, ha sido objeto de múltiples evaluaciones, lo que permite visualizar cambios sustanciales en la labor docente, tal es así que, se convierte en una alternativa en la labor pedagógica del docente, en donde los docentes ya no estarán desatendidos en sus necesidades pedagógicas, sino por el contrario tendrán el soporte necesario para enfrentar situaciones que limiten su accionar pedagógico. Por otro lado, el acompañamiento al docente implica que, además de recibir el soporte pedagógico, estará permanentemente orientado, con una estrategia formativa que le permita reflexionar sobre su práctica para la toma de decisiones de mejora (Balarín et al., 2016). 
La Gerencia Regional de Educación La Libertad (GRELL, 2019), reporta que, en la Unidad de Gestión Educativa Local de Santiago de Chuco, se debe continuar con el programa de acompañamiento pedagógico debido a que, se debe seguir fortaleciendo las competencias pedagógicas de los docentes, incorporando, a su práctica de enseñanza, estrategias y recursos, que les permita responder a las necesidades del contexto, considerando el nuevo desafío de la virtualidad y la forma didáctica de generar aprendizajes en esta modalidad. Existe un escaso manejo de los recursos electrónicos, sumado a la carencia de dispositivos virtuales, genera como consecuencia, la dificultad para adaptarse a esta nueva forma remota de aprendizaje.

El presente estudio, en relación a la justificación, se tomará en consideración lo afirmado por, (Hernández et al., 2014), un estudio se orienta en su relevancia e importancia por diversas causales, una de estas es que una investigación propone alternativas que permiten resolver una situación cotidiana en el aprendizaje de los estudiantes, otra posibilidad de la relevancia de un estudio, es que aporta al marco teórico, enriqueciendo la literatura científica, otro aspecto que le otorga relevancia a un estudio, es cuando aporta al método científico, es decir, el estudio se convierte en un antecedente para otras investigaciones de similares características; por ejemplo, brinda un instrumento para el recojo de datos que permita observar una variable de estudio, además, brinda un procedimiento nuevo en la metodología científica.

En el presente estudio, para la selección de los antecedentes, se consideró los trabajos con similares características. Así tenemos, en el ámbito internacional, se consideró como ejemplo el estudio realizado por, (Mairena, 2016), quien realizó un estudio, el cual estuvo orientado a comprobar la relación entre sobre seguimiento pedagógico y desempeño docente. El estudio fue de enfoque cuantitativo con alcance relacional, la muestra estuvo integrada por cuarenta docentes noveles en una universidad, a quienes se les aplicó como instrumento un cuestionario, en quienes se demostró que, el seguimiento pedagógico no se relaciona con el desempeño en el contexto de los sujetos que participaron en la investigación. Estos hallazgos reflejan que, el acompañamiento realizado a los docentes no guarda relación con su desempeño, es decir que el desempeño que demuestran los docentes en su práctica pedagógica no está influenciado por acompañamiento que reciben, probablemente sean otros factores los que influyen como el entorno educativo en 
el que desempeña su labor, el apoyo de parte de los padres de familia, el clima escolar, el cual determina el ambiente saludable de trabajo.

Así mismo, se consideró el estudio realizado por (Valle, 2016), quien realizó una investigación sobre el acompañamiento en la mejora del desempeño docente. El estudio, se orientó bajo el propósito de valorar el seguimiento docente en la mejora de su desempeño. El estudio fue de enfoque cuantitativo, con un alcance descriptivo correlacional. La unidad de análisis estuvo integrada por veintisiete docentes de primaria de la región de Managua, el instrumento aplicado fue un cuestionario de treinta ítems, en donde los hallazgos demostraron que, el seguimiento docente no guarda relación con su desempeño, generado por un escaso acompañamiento a la práctica docente de quienes lideran la institución académica. Estos resultados demuestran que, si no se brinda un seguimiento sistemática y permanente a la práctica pedagógica con el propósito de brindar el soporte que necesitan, el desempeño de los docentes se evidenciará en una limitación, repercutiendo en su rendimiento profesional y en su desempeño laboral. Es importante brindar las herramientas necesarias y el soporte consistente al cuerpo docente, porque esta acción repercutirá en su desempeño profesional.

En el ámbito nacional, se consideró como antecedente, el trabajo realizado por, (Aguirre, 2019), quien realizó un estudio sobre el seguimiento a la práctica pedagógica y su desempeño. El estudio se orientó con el propósito de comprobar la relación entre el seguimiento pedagógico y el desempeño profesional del docente. La investigación fue de enfoque cuantitativa, con un nivel descriptivo relacional. Los sujetos de investigación fueron treinta docentes del nivel secundaria, a quienes se les aplicó dos cuestionarios con escala de medición ordinal. Los hallazgos determinaron que, el seguimiento docente se relaciona con el desempeño profesional de los docentes, estos resultados reflejan que el soporte pedagógico brindado al cuerpo docente tiene una relación significativa con su desempeño en la práctica profesional, por lo tanto, es muy importante brindar el apoyo permanente y sistemático a los docentes para mejorar su desempeño profesional.

En el ámbito local, se consideró como antecedente, el trabajo realizado por, (Torres, 2020), quien realizó un estudio sobre acompañamiento pedagógico y su relación con el desempeño profesional de los docentes en instituciones educativas de Trujillo. El estudio fue orientado con el propósito de comprobar la relación que existe entre el seguimiento a la práctica pedagógica con el desempeño profesional de los docentes. El estudio fue una 
investigación de enfoque cuantitativo, con alcance relacional. Los sujetos que intervinieron en la investigación fueron veintinueve docentes, a quienes se les aplicó como instrumentos dos cuestionarios con escala de medición ordinal. Los hallazgos demostraron que, el seguimiento a la práctica pedagógica se relaciona significativamente con el desempeño profesional de los docentes que conformaron la unidad de análisis en este estudio. Estos resultados significan que, si los docentes del contexto en que se realizó la investigación, reciben el soporte necesario para mejorar su desempeño laboral, esto influye directamente sobre su práctica pedagógica

Las teorías científicas, en relación a las variables de estudio, le otorgan la consistencia fundamentada, tal es así que, en relación al acompañamiento pedagógico, tenemos a (Bismar, 2018), quien define el acompañamiento a la práctica docente como el proceso de diagnosticar y atender las necesidades de los docentes, es decir que refleja cuales son las fortalezas pero principalmente las debilidades que presentan los profesionales en educación, así mismo, se entiende el acompañamiento como el seguimiento y monitoreo a la acción del docente, lo cual permite sobre la reflexión y autoevaluación proponer alternativas de mejora.

Así mismo, (Ágreda y Pérez, 2020), sostienen que, el acompañamiento a la práctica pedagógica, es un proceso que tiene como propósito brindar el soporte necesario a la práctica docente y que esta acción permita implementar acciones de mejora en la praxis docente. Este proceso necesita de una verdadera interacción entre el acompañante y el acompañado, en donde se brinde y genere relaciones interpersonales saludables basadas en el respeto y la consideración para una auténtica reflexión y mejora del desempeño profesional.

Las teorías científicas, en relación a la variable desempeño docente, tenemos a (Guevara y Martínez, 2016), sostienen que, el desempeño es la acción docente que guarda relación con las responsabilidades y vocación de servicio del docente. Por otro lado, el desempeño del docente es el reflejo de su práctica pedagógica, la cual se evidencia en sintonía de su formación profesional, es por ello que un docente que carece o presenta dificultades en su formación profesional, tendrá dificultades para ejercer su labor profesional, es por ello que, el docente necesita el soporte necesario dentro de su formación en servicio para consolidar sus competencias profesionales y poderlas al servicio de sus comunidades educativas. 
En coherencia a lo anterior, (Cuesta, 2019), sustenta que, el desempeño docente es el cumplimiento de las funciones profesionales relacionadas a la formación pedagógica y a la vocación de servicio de la docencia. El desempeño responde a la formación y vocación del docente, es por ello que, si la formación y vocación presentan carencias, lo más probable que estos puntos críticos se evidencien en el desempeño, por otro lado, si la formación y vocación está debidamente consolidada esto se observará en el desempeño profesional. Es por ello que, el Estado a través de políticas públicas debe implementar programas que brinden un acompañamiento y seguimiento a la práctica profesional docente

Teniendo en cuenta las consideraciones mostradas anteriormente, el problema general de esta investigación se planteó de la siguiente manera: ¿Cuál es la influencia del acompañamiento pedagógico en el desempeño docente en la Unidad de Gestión Educativa Local de Santiago de Chuco, 2020? Por otro lado, el presente estudio, plantea el siguiente objetivo general: determinar la influencia del acompañamiento pedagógico en el desempeño docente en la Unidad de Gestión Educativa Local de Santiago de Chuco, 2020. Finalmente, se formula la siguiente hipótesis general: el acompañamiento pedagógico influye significativamente en el desempeño docente en la Unidad de Gestión Educativa Local de Santiago de Chuco, 2020.

\section{MÉTODO}

El estudio, según su enfoque, es cuantitativo porque se apoyará en la estadística descriptiva e inferencial en recojo, procesamiento e interpretación de los datos. Según su finalidad, es aplicada, porque bridará información relevante, la cual será el insumo para proponer alternativas de mejora en beneficio de una entidad profesional como la docencia. Así mismo, presenta un diseño no experimental transversal descriptivo correlacional causal, porque se orienta con el objetivo de determinar la influencia de una variable de estudio sobre la otra en un contexto determinado (Hernández et al., 2014). El esquema es el siguiente:

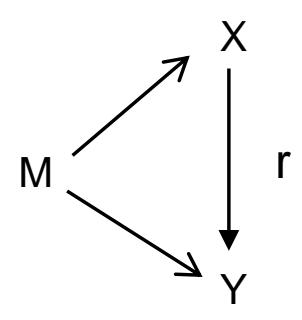




\section{Dónde:}

\section{M: Docentes}

$\mathrm{X}$ : Acompañamiento pedagógico

Y: Desempeño docente

r: Relación causal de X sobre Y.

La población muestra objeto de estudio del presente trabajo estuvo comprendido por 24 docentes de la UGEL Santiago de Chuco.

\section{RESULTADOS}

A continuación, se presenta los resultados de la variable 1, acompañamiento pedagógico en la Unidad de Gestión Educativa Local de Santiago de Chuco, 2020, los cuales se visualizan en la siguiente tabla.

Tabla 1. Acompañamiento pedagógico en la Unidad de Gestión Educativa Local de Santiago de Chuco, 2020.

\begin{tabular}{lcc}
\hline Niveles de valoración & ni & \% \\
\hline Alto & 6 & 25.0 \\
\hline Medio & 17 & 70.8 \\
\hline Bajo & 1 & 4.2 \\
\hline Total & 24 & 100.0 \\
\hline
\end{tabular}

Fuente: Base de datos del cuestionario acompañamiento docente.

En la tabla 1, en relación al acompañamiento pedagógico en la Unidad de Gestión Educativa Local de Santiago de Chuco, se puede observar que, $25.0 \%$ presenta un nivel alto, $70.8 \%$ presenta un nivel medio, $4.2 \%$ presenta un nivel bajo. Predominando el nivel medio en el acompañamiento pedagógico en la Unidad de Gestión Educativa Local de Santiago de Chuco, 2020.

Veamos ahora la siguiente variable.

Tabla 2. Desempeño docente en la Unidad de Gestión Educativa Local de Santiago de Chuco, 2020.

\begin{tabular}{lcc}
\hline Niveles de valoración & ni & \% \\
\hline Alto & 13 & 54.2 \\
\hline Medio & 11 & 45.8 \\
\hline Bajo & 0 & 0.0 \\
\hline Total & 24 & 100.0 \\
\hline
\end{tabular}

Fuente: Base de datos del cuestionario desempeño docente. 
En la tabla 2, en relación al desempeño docente en la Unidad de Gestión Educativa Local de Santiago de Chuco, se puede observar que, 54.2\% presenta un nivel alto, $45.8 \%$ presenta un nivel medio, $0.0 \%$ presenta un nivel bajo. Predominando el nivel alto en el desempeño docente en la Unidad de Gestión Educativa Local de Santiago de Chuco, 2020.

Hipótesis: El acompañamiento pedagógico influye significativamente en el desempeño docente en la Unidad de Gestión Educativa Local de Santiago de Chuco, 2020.

Nivel de significancia o riesgo: $\alpha=0,05$.

Tabla 3. Influencia del acompañamiento pedagógico en el desempeño docente en la Unidad de Gestión Educativa Local de Santiago de Chuco, 2020.

\begin{tabular}{|c|c|c|c|c|c|c|c|c|}
\hline \multirow{3}{*}{ Desempeño docente } & \multicolumn{6}{|c|}{ Acompañamiento pedagógico } & \multirow{2}{*}{\multicolumn{2}{|c|}{ Total }} \\
\hline & \multicolumn{2}{|c|}{ Alto } & \multicolumn{2}{|c|}{ Medio } & \multicolumn{2}{|c|}{ Bajo } & & \\
\hline & ni & $\%$ & ni & $\%$ & ni & $\%$ & ni & $\%$ \\
\hline Alto & 2 & 8.3 & 10 & 41.7 & 1 & 4.2 & 13 & 54.2 \\
\hline Medio & 4 & 16.7 & 7 & 29.2 & 0 & 0.0 & 11 & 45.8 \\
\hline Bajo & 0 & 0.0 & 0 & 0.0 & 0 & 0.0 & 0 & 0.0 \\
\hline Total & 6 & 25.0 & 17 & 70.8 & 1 & 4.2 & 24 & 100.0 \\
\hline
\end{tabular}

\begin{tabular}{|c|c|c|c|c|}
\hline \multicolumn{5}{|c|}{ Prueba correlacional causal } \\
\hline & & & $\begin{array}{c}\text { Acompañamiento } \\
\text { pedagógico }\end{array}$ & $\begin{array}{c}\text { Desempeño } \\
\text { docente }\end{array}$ \\
\hline \multirow{6}{*}{$\begin{array}{l}\text { Rho de } \\
\text { Spearman }\end{array}$} & \multirow{3}{*}{$\begin{array}{l}\text { Acompañamiento } \\
\text { pedagógico }\end{array}$} & $\begin{array}{l}\text { Coeficiente de } \\
\text { correlación }\end{array}$ & 1,000 &,- 282 \\
\hline & & Sig. (bilateral) & . &, 183 \\
\hline & & $\mathrm{N}$ & 24 & 24 \\
\hline & \multirow{3}{*}{$\begin{array}{l}\text { Desempeño } \\
\text { docente }\end{array}$} & $\begin{array}{l}\text { Coeficiente de } \\
\text { correlación }\end{array}$ &,- 282 & 1,000 \\
\hline & & Sig. (bilateral) &, 183 & . \\
\hline & & $\mathrm{N}$ & 24 & 24 \\
\hline
\end{tabular}

En la tabla 3, en relación a la prueba de hipótesis general, el estadístico de Rho de Spearman, demuestra que, (p-valor: 0,183>0,010), en consecuencia, se acepta la hipótesis nula (Ho) y se rechaza la hipótesis alterna $(\mathrm{Hi})$, se concluye que, el acompañamiento pedagógico no influye significativamente en el desempeño docente en la Unidad de Gestión Educativa Local de Santiago de Chuco, 2020. 
Decisión estadística: Puesto que (p-valor: 0,183>0,010 y rho=-0,282), en consecuencia, se acepta la hipótesis nula.

Conclusión estadística: Se concluye que, el acompañamiento pedagógico no influye significativamente en el desempeño docente en la Unidad de Gestión Educativa Local de Santiago de Chuco, 2020.

\section{DISCUSIÓN}

Estadísticamente se tiene que (p-valor: 0,183>0,010 y rho=-0,282), en consecuencia, se acepta la hipótesis nula (Ho) y se rechaza la hipótesis alterna (Hi), se concluye que, el acompañamiento pedagógico no influye significativamente en el desempeño docente en la Unidad de Gestión Educativa Local de Santiago de Chuco, 2020.

Sin duda este antecedente coincide con los resultados obtenidos entre estas dos variables, ya que, (Mairena, 2016), en su investigación, Relación entre seguimiento pedagógico y desempeño docente en la región de Managua, donde concluye que, el seguimiento pedagógico no se relaciona con el desempeño en el contexto de los sujetos que participaron en la investigación. Estos hallazgos reflejan que, el acompañamiento realizado a los docentes no guarda relación con su desempeño, es decir que el desempeño que demuestran los docentes en su práctica pedagógica no está influenciado por acompañamiento que reciben, probablemente sean otros factores los que influyen como el entorno educativo en el que desempeña su labor, el apoyo de parte de los padres de familia, el clima escolar, el cual determina el ambiente saludable de trabajo.

Los resultados obtenidos también son fortalecidos, en la medida que la teoría del acompañamiento pedagógico, tenemos a (Bismar, 2018), nos dice que, el acompañamiento a la práctica docente es el proceso de diagnosticar y atender las necesidades de los docentes, es decir que refleja cuales son las fortalezas pero principalmente las debilidades que presentan los profesionales en educación, así mismo, se entiende el acompañamiento como el seguimiento y monitoreo a la acción del docente, lo cual permite sobre la reflexión y autoevaluación proponer alternativas de mejora.

Así mismo, también tenemos los hallazgos de (Valle, 2016), quien, en su investigación, El acompañamiento en la mejora del desempeño docente de primaria de la región de Managua, donde concluye que, el seguimiento docente no guarda relación con su desempeño, generado por un escaso acompañamiento a la práctica docente de quienes lideran la institución académica. Estos resultados demuestran que, si no se brinda un 
seguimiento sistemática y permanente a la práctica pedagógica con el propósito de brindar el soporte que necesitan, el desempeño de los docentes se evidenciará en una limitación, repercutiendo en su rendimiento profesional y en su desempeño laboral. Es importante brindar las herramientas necesarias y el soporte consistente al cuerpo docente, porque esta acción repercutirá en su desempeño profesional.

Los resultados guardan también una correspondencia biunívoca de igual manera con la teoría sobre el acompañamiento a la práctica pedagógica, tenemos a (Ágreda y Pérez, 2020), quienes sostienen que, el acompañamiento a la práctica pedagógica, es un proceso que tiene como propósito brindar el soporte necesario a la práctica docente y que esta acción permita implementar acciones de mejora en la praxis docente. Este proceso necesita de una verdadera interacción entre el acompañante y el acompañado, en donde se brinde y genere relaciones interpersonales saludables basadas en el respeto y la consideración para una auténtica reflexión y mejora del desempeño profesional.

\section{CONCLUSIONES}

1) Se ha determinado que, el acompañamiento pedagógico no influye significativamente en el desempeño docente en la Unidad de Gestión Educativa Local de Santiago de Chuco, 2020.

2) Se ha determinado que, predominó el nivel alto en la dimensión ámbito personal en el acompañamiento pedagógico en la Unidad de Gestión Educativa Local de Santiago de Chuco, 2020.

3) Se ha determinado que, predominó el nivel alto en la dimensión ámbito pedagógico didáctico en el acompañamiento pedagógico en la Unidad de Gestión Educativa Local de Santiago de Chuco, 2020.

4) Se ha determinado que, predominó el nivel alto en la dimensión desarrollo profesional en el acompañamiento pedagógico en la Unidad de Gestión Educativa Local de Santiago de Chuco, 2020.

5) Se ha determinado que, predominó el nivel alto en vínculo de la comunidad en el acompañamiento pedagógico en la Unidad de Gestión Educativa Local de Santiago de Chuco, 2020.

6) Se ha determinado que, predominó el nivel alto en la dimensión clima propicio para el aprendizaje en el desempeño docente en la Unidad de Gestión Educativa Local de Santiago de Chuco, 2020. 
7) Se ha determinado que, predominó el nivel alto y medio en la dimensión proceso de enseñanza en el desempeño docente en la Unidad de Gestión Educativa Local de Santiago de Chuco, 2020.

8) Se ha determinado que, predominó el nivel alto en la dimensión evaluación del aprendizaje en el desempeño docente en la Unidad de Gestión Educativa Local de Santiago de Chuco, 2020.

\section{REFERENCIAS BIBLIOGRÁFICAS}

Ágreda, A., y Pérez, M. (2020). Relationship between pedagógica! accompaniment and reflective teaching practice. Scielo, 50-62.

Aguirre, A. (2019). El acompañamiento pedagógico y el desempeño docente de la Institución Educativa № 14793 “Luciano Castillo Colonna”, Bellavista, Sullana, 2019. (Tesis posgrado). Universidad César Vallejo, Sullana.

Baena, G. (2017). Metodología de la investigación. México: PATRIA.

Balarín, M., Rodríguez, M., y Escudero, A. (2016). Evaluación del diseño y proceso de implementación de la intervención de Soporte Pedagógico del Ministerio de Educación del. Lima: GRADE - FORGE.

Bismar, G. (2018). Estrategias de acompañamiento pedagógico para el desarrollo profesional docente. Revista caribeña de investigación educativa., 24-52.

Cuenca, R. (2015). Las carreras docentes en América Latina: la acción meritocrática para el desarrollo profesional. Santiago de Chile: UNESCO-OREALC.

Cuesta, A. (2019). Manual para la evaluación del desempeño laboral. Madrid: MARCOMBO.

GRELL. (17 de abril de 2019). Gerencia Regional de Educación La Libertad. Obtenido de Programas presupuestales: http://grell.gob.pe/

Guevara, A., y Martínez, G. (2016). Evaluación del desempeño docente. Redalyc, 113124.

Hernández, R., Fernández, C., \& Baptista, P. (2014). Metodología de la investigación científica. México D.F: Mc GRAW-HILL.

Mairena, E. (2016). Acompañamiento pedagógico y desempeño de los docentes noveles en los departamentos de física y tecnología educativa de la facultad de educación e idiomas. (Tesis de posgrado). Universidad Nacional Autónoma de Nicaragua, Managua, Nicaragua. 
Rivas, A. (2015). América Latina después de PISA: Lecciones aprendidas de la educación en siete países (2000-2015). Buenos Aires: Fundación CIPPEC.

Torres, E. (2020). Acompañamiento pedagógico y desempeño docente en I.I.E.E. por convenio del distrito de Salaverry en Trujillo, 2020. (Tesis de posgrado). Universidad César Vallejo, Trujillo, Perú.

UNESCO. (2019). Organización de las Naciones Unidas para la Educación, la Ciencia y la Cultura. París: GRADE.

Valle, I. (2016). Incidencia del acompañamiento pedagógico para la mejora del desempeño docente de educación primaria en el Instituto Loyola, ubicado en el departamento y municipio de Managua, distrito I, durante el periodo del año 2015. (Tesis de pos grado). Universidad Nacional Autónoma de Nicaragua, Managua, Nicaragua. 